\title{
El Aprendizaje basado en Problemas como Estrategia para el Desarrollo de Competencias Específicas en Estudiantes de Ingeniería
}

\author{
Flavio H. Fernández y Julio E. Duarte \\ Universidad Pedagógica y Tecnológica de Colombia, Facultad Seccional Duitama. Grupo de Didáctica para \\ la Enseñanza de la Ciencia y la Tecnología en Niños, DECTEN. Carrera 18 Calle 23. Duitama, Boyacá- \\ Colombia (e-mail: flaviofm1@gmail.com; julioenriqued1@gmail.com)
}

Recibido May. 16, 2013; Aceptado Jun. 20, 2013; Versión final recibida Ago. 06, 2013

\begin{abstract}
Resumen
El trabajo reporta la aplicación de la metodología de aprendizaje basado en problemas adaptada al desarrollo de competencias específicas en estudiantes de ingeniería. La propuesta considera tres etapas para su aplicación: sensibilización, seguimiento y evaluación. Se realizó una prueba piloto con estudiantes de octavo semestre de ingeniería electromecánica de la Universidad Pedagógica y Tecnológica de Colombia, quienes desarrollaron un proyecto a partir de una problemática real. Los resultados evidencian mejoras en las competencias relacionadas con la solución de problemas del mundo real así como en la gestión de proyectos a través de la elaboración y socialización de informes. Se detectaron también algunas falencias en los conocimientos disciplinares. Por lo que la metodología propuesta puede ser utilizada como una herramienta para diagnosticar y corregir las debilidades en el proceso formativo de los futuros ingenieros.
\end{abstract}

Palabras clave: aprendizaje basado en problemas, formación de ingenieros, ingeniería electromecánica, competencias específicas

\section{Problem-Based Learning as Strategy to Develop Specific Skills in Engineering Students}

\begin{abstract}
This paper reports the application of the problem-based learning methodology adapted to the development of specific skills in engineering students. This proposal considers three stages in its application: sensibilization, following up and evaluation. The study was applied to students of the eighth semester of electromechanical engineering at Pedagogical and Technological University of Colombia, who developed a project, based on a real situation. The results provide evidence of improvement in skills related to the solution of real world problems as well as to project management through the preparation and socialization of reports. Also, some shortcomings were detected in reference to the disciplinary knowledge. Therefore, the proposed methodology can be used as a tool for diagnosing and correcting weaknesses in the formative process of the future engineers.
\end{abstract}

Keywords: problem-based learning, engineering education, electromechanical engineering, specific skills 


\section{INTRODUCCIÓN}

La formación del ingeniero hoy en día es un desafío debido a la gran cantidad de información disponible, a la complejidad creciente de los problemas a los que se deben enfrentar y a la globalización de los mercados. Además, el medio ambiente se ha convertido en un factor fundamental en la actividad del ingeniero por la necesidad de conseguir productos y procesos sustentables que no lo deterioren; igualmente, es importante la responsabilidad social que asuma el profesional frente a los productos generados por las nuevas tecnologías y su impacto en todos los ámbitos de la actividad humana. Además, las estructuras corporativas son cada vez más participativas, exigiéndole al profesional más trabajo en grupo y responsabilidad en la toma de decisiones (Rugarcia et al., 2000; Regalado et al., 2010). Lo anterior implica que un profesional idóneo debe desarrollar gran capacidad de adaptación al cambio, unida al manejo adecuado de la información, con una actitud ética que le permita tomar decisiones adecuadas al entorno socio-cultural en el cual se desenvuelve.

Existe una preocupación constante de universidades y docentes, los cuales buscan desarrollar y adaptar nuevas estrategias pedagógicas y didácticas que permitan la formación de profesionales de la ingeniería con las competencias exigidas por entornos laborales y sociales cada vez más dinámicos. Las nuevas metodologías buscan potenciar el desarrollo de competencias genéricas como: el aprender a aprender, organizar y planificar, analizar y sintetizar, aplicar los conocimientos a la práctica, expresarse con claridad de manera oral y escrita en la propia lengua, capacidad crítica y autocrítica, trabajar de forma colaborativa, capacidad de iniciativa y liderazgo y conocer una segunda lengua (Galvis, 2007; Schmal, 2012). Igualmente se busca el desarrollo de competencias específicas, las cuales dependerán de las áreas de conocimiento propias del programa académico que se esté considerando. Estos aspectos han sido recogidos en los criterios para la acreditación de programas de ingeniería en las diversas instituciones responsables de éste proceso, como el ABET (Acreditation Board for Engineering and Technology) en los Estados Unidos (Rugarcia et al., 2000).

Entre las diversas metodologías para desarrollar las competencias se pueden mencionar: el aprendizaje cooperativo, el aprendizaje colaborativo, el aprendizaje basado en competencias, el aprendizaje orientado por proyectos y el aprendizaje basado en problemas, entre otras (Nascimento y Amaral, 2012; Moreno et al., 2007; Regalado et al., 2011; Hernández, 2010; Benítez y García, 2013; Lehmanna et al., 2008). Estas metodologías se han visto favorecidas con el uso de las Tecnologías de la Información y la Comunicación, TIC, las cuales, en el caso de la ingeniería, implican la utilización de plataformas en internet para el desarrollo de actividades de formación, laboratorios virtuales y experimentación remota, interfaces web para visualizar contenidos, junto con herramientas de simulación específicamente diseñadas para desarrollar habilidades y destrezas en los futuros ingenieros (Vacca et al., 2011; Alejandro, 2004; Ertugrul, 2000; Okutsu et al., 2013).

Existen diversas metodologías que buscan acercar al estudiante a la solución de problemas del mundo real. Una de ellas es el Aprendizaje Basado en Problemas, ABP, la cual permite centrar el aprendizaje en el estudiante, e introducir en la enseñanza problemas abiertos y más próximos a su desempeño profesional (ITESM, 2000; Restrepo, 2005). Esta metodología favorece la posibilidad de interrelacionar distintas materias o disciplinas académicas para intentar solucionar un problema, ya que los alumnos necesitan recurrir a conocimientos adquiridos en diversas asignaturas. Esto permite la integración de conocimientos, desarrollando así la competencia del saber hacer en contexto.

En el presente trabajo se reporta la experiencia vivida con la aplicación de la metodología ABP, adaptada para el desarrollo de competencias profesionales en los estudiantes de Ing. Electromecánica, de la Universidad Pedagógica y Tecnológica de Colombia. El documento inicia presentando los conceptos básicos del ABP y el de las competencias. Igualmente se describe la problemática que dio origen al proyecto y se presenta la metodología utilizada con estudiantes de octavo semestre. Como resultado se presentan las apreciaciones de los estudiantes y el docente que participaron en la experiencia, junto con los cambios detectados en el desarrollo de competencias profesionales. Finalmente se reportan las conclusiones de aplicar el ABP y se brindan algunas recomendaciones que permitirán optimizar la implantación de este tipo de metodologías.

\section{EL APRENDIZAJE BASADO EN PROBLEMAS, ABP}

Hace más de sesenta años, las escuelas de medicina de los Estados Unidos pusieron en duda la eficiencia de los modelos curriculares de dos etapas tradicionalmente utilizados en sus estudios: la primera etapa estaba centrada en estudios intensivos en ciencias básicas y la segunda en la realización de estudios clínicos exhaustivos. Los profesores de la facultad de medicina se convencieron de que dicho modelo constituía una forma ineficiente e inhumana de preparar a los futuros médicos, dado el 
descomunal aumento de la información médica, de las innovaciones tecnológicas y de las demandas constantes de cambios en las prácticas médicas. Por ello propusieron un método diferente, fundamentado en una estrategia conocida como Aprendizaje Basado en Problemas, ABP, concebida para integrar el proceso de enseñanza mediante la confrontación con situaciones reales de la práctica de la medicina (Iglesias, 2002).

El ABP es uno de los métodos de enseñanza - aprendizaje que ha tenido mayor aceptación entre las instituciones de educación superior en los últimos años. Es así como el ABP ha sido utilizado como una estrategia general a lo largo del plan de estudios de una carrera profesional, implementado como estrategia de trabajo a lo largo de cursos específicos, e incluso como una técnica didáctica aplicada para la revisión de ciertos objetivos de aprendizaje (Echazarreta y Haudemand, 2009; Rosado et al., 2008; Painean et al., 2012; Kurt y Ayas, 2012). Los impulsores del ABP parten de la base de que los estudiantes obtienen conocimiento en cada experiencia de aprendizaje y consideran que hay mejores posibilidades de aprender cuando se cumplen las siguientes condiciones: el conocimiento previo es activado y alentado para incorporar nuevos conocimientos, se dan numerosas oportunidades para aplicarlos y el aprendizaje de nuevos conocimientos se produce en el contexto en que se utilizará posteriormente (Iglesias, 2002). Mientras que en el método tradicional primero se expone la información y posteriormente se busca su aplicación en la resolución de un problema, en el caso del ABP primero se presenta el problema, se identifican las necesidades de aprendizaje, se busca la información necesaria y, finalmente, se regresa al problema.

En el recorrido que viven los estudiantes desde el planteamiento inicial del problema hasta su solución, ellos trabajan de manera colaborativa en pequeños grupos, compartiendo su experiencia de aprendizaje. Además, se tiene la posibilidad de desarrollar habilidades como observar el entorno, reflexionar y comunicar ideas, y poner en práctica actitudes y valores que en el método convencional expositivo difícilmente se pueden explicitar (Domínguez et al., 2008). El ABP es un método de enseñanza caracterizado por el uso de problemas del "mundo real" establecidos como contextos en los que los estudiantes desarrollan su capacidad crítica y de solución de problemas, al tiempo que adquieren los conceptos esenciales de un determinado ámbito de conocimiento.

Una de las ventajas del ABP, que llevaron a elegirlo como estrategia pedagógica para el desarrollo de competencias específicas en los futuros ingenieros electromecánicos, está el hecho de que lleva a los estudiantes al aprendizaje de los contenidos de información de manera similar a la que utilizarán en situaciones futuras, fomentando que lo aprendido se comprenda y no solo se memorice. Igualmente se tuvo en cuenta que el ABP permite que el conocimiento de varias disciplinas se integre para dar solución al problema sobre el cual se está trabajando, de tal modo que el aprendizaje no se da en fracciones sino de una manera integral y dinámica.

\section{Competencias}

El término de competencias ha venido evolucionando desde su uso inicial en el ámbito empresarial con el llamado enfoque centrado en la tarea, en el cual surgen las llamadas competencias laborales; éstas se describen como la capacidad efectiva para llevar a cabo exitosamente una actividad laboral plenamente identificada (González y Ramírez, 2011). En esta definición se enfatizan los conocimientos, habilidades y destrezas que debe poseer una persona para cumplir eficientemente con una tarea determinada.

En el enfoque centrado en el perfil ocupacional, se establecen las competencias profesionales, las cuales pueden verse como el resultado de un proceso de educación de la personalidad para el desempeño profesional, eficiente y responsable, que no culmina con el egreso del estudiante de un centro de formación profesional sino que lo acompaña durante el proceso de su desarrollo profesional, en el ejercicio de la profesión (González, 2002). Desde esta perspectiva, lo importante no es la posesión de determinados conocimientos, sino el uso que se haga de ellos, que se tenga motivación para hacerlo y compromiso para alcanzar un resultado.

Otra forma de entender a las competencias es como desempeños integrales para interpretar, argumentar y resolver problemas del contexto con creatividad, idoneidad, mejoramiento continuo y ética, desarrollando y poniendo en acción de forma articulada el saber ser, el saber convivir, el saber hacer y el saber conocer (Tobón y Jayk, 2012). Surge así el modelo de competencias profesionales integrales, el cual las organiza en tres niveles: básicas, genéricas y específicas, cuyo rango va de lo general a lo particular. Las competencias básicas son las capacidades intelectuales indispensables para el aprendizaje de una profesión; en ellas se encuentran las competencias cognitivas, técnicas y metodológicas, muchas de las cuales son adquiridas en los niveles educativos previos (por ejemplo el uso adecuado de los lenguajes oral, escrito y matemático). Las competencias genéricas son la base común de la profesión o se refieren a las 
situaciones concretas de la práctica profesional que requieren de respuestas complejas. Por último, las competencias específicas son la base particular del ejercicio profesional y están vinculadas a condiciones específicas de ejecución (González y Ramírez, 2011).

\section{Planteamiento del problema}

La Universidad Pedagógica y Tecnológica de Colombia, UPTC, en la Facultad Seccional Duitama, Boyacá, Colombia, cuenta desde hace 33 años con el programa de ingeniería electromecánica, de modalidad presencial, con una duración de 10 semestres y otorga el título de INGENIERO ELECTROMECÁNICO. En el proceso de autoevaluación del programa con fines de acreditación, realizado entre los años 2003 a 2004, se identificó como problema la poca incidencia de los futuros ingenieros en la solución de problemas del entorno. Esta problemática no es exclusiva de la ingeniería en Colombia y Latinoamérica, sino que también es una preocupación muy sentida en Europa, donde se han venido tomando correctivos a partir de la declaración de Bolonia (Mesa et al., 2008).

Para su desarrollo, el plan de estudios contempla la realización de proyectos aplicados, que se ejecutarán paralelamente con las asignaturas del campo de formación específico profesional, pero no contempla ninguna estrategia pedagógica que le brinde al estudiante las herramientas necesarias para desarrollar estas competencias. En los últimos años esta debilidad se ha venido subsanando a través del desarrollo de proyectos de grado y pasantías, donde el futuro egresado busca, evalúa, desarrolla y soluciona problemas del entorno a nivel industrial y empresarial. Sin embargo, esta actividad se cumple demasiado tarde, al finalizar las asignaturas, limitando el desarrollo de competencias profesionales de forma integral. En este sentido, se observa la necesidad de implementar una estrategia formativa que aborde las debilidades descritas anteriormente, tendientes a buscar un aprendizaje significativo que permita a los estudiantes aplicar de manera integral los conocimientos recibidos durante su formación.

\section{METODOLOGÍA PROPUESTA}

A continuación se describe la metodología ABP propuesta para el desarrollo de las siguientes competencias contempladas en los planes de estudio de los programas de ingeniería:

C1: Proponer la solución de problemas del entorno a través de proyectos de ingeniería.

C2: Conocer los métodos y estrategias más adecuadas para la recolección, manejo e interpretación de la información y el desarrollo de proyectos en ingeniería.

C3: Elaborar informes, parciales y finales, y socializar los resultados generados a partir de la gestión de proyectos de ingeniería.

\section{Primer Momento: Sensibilización}

En este se describe la metodología a los estudiantes, se presentan los problemas, se constituye el grupo y se selecciona el problema. También se describen, de forma clara, los compromisos y el papel de los actores involucrados en la experiencia.

\section{Segundo Momento: Seguimiento a la Solución del Problema}

Para identificar los avances del proyecto que se desarrolla a lo largo del semestre, se proponen cuatro etapas o puntos de control, así: anteproyecto, informe de avances, informe técnico de la solución e informe final:

- Anteproyecto: En esta etapa el grupo elaborará un documento que dé cuenta de la identificación del problema, de las hipótesis de trabajo y de la metodología prevista junto con los recursos necesarios para resolverlo.

- Informe de avances: El grupo realizará un informe escrito de los avances en el desarrollo del proyecto, de modo que se puedan establecer el porcentaje de cumplimiento en las actividades, dificultades y logros parciales.

- Informe técnico de la solución: El grupo realizará un informe, desde el punto de vista técnico, en el cual se dé cuenta de las alternativas para la solución del problema, justificando las decisiones tomadas, desde el punto de vista ingenieril.

- Informe final: Como resultado del proyecto desarrollado, al terminar el semestre, se espera disponer del informe final, representado en un artículo con los elementos propios de una revista científica. 


\section{Tercer Momento: Evaluación y Mecanismos}

Como mecanismos de evaluación se establecieron el informe escrito y la presentación oral. Se elaborarán cuatro informes durante el curso, cada uno correspondiente a cada etapa del desarrollo del trabajo: anteproyecto, informe de avances, informe técnico de la solución e informe final, cuyas características se describieron en el apartado anterior. Los criterios de evaluación para el informe escrito se dividen en dos categorías: de fondo y de forma. En los primeros se consideran: que se ilustren los métodos o procedimientos, que se evidencie el trabajo realizado y el grado de solución del problema. En los criterios de forma se consideran: la presentación y aplicación de las normas asociadas a cada tipo de documento, coherencia y claridad en la redacción y que se emplee vocabulario pertinente a la temática desarrollada.

En cada fase habrá una presentación oral sustentando la información contenida en el informe escrito. En los criterios de evaluación para la sustentación oral se consideran: claridad en la exposición, seguridad del expositor, que la presentación refleje el contenido del documento escrito, uso adecuado del vocabulario técnico, uso de medios audiovisuales y presentación personal.

Se sugiere una escala valorativa de uno (1.0) a cinco (5.0) para cada uno de los criterios anteriores. Además se le permitirá al estudiante la posibilidad de analizar el desempeño de sí mismo, reflejado en la presentación oral (Autoevaluación); evaluar el proceso realizado por sus compañeros, reflejado en la presentación oral (Coevaluación). El informe escrito y la presentación oral serán los mecanismos de evaluación disponibles para el docente (Heteroevaluación). Para la evaluación se sugieren los siguientes rubros: autoevaluación 25\%, coevaluación 25\% y heteroevaluación 50\%; lo anterior teniendo en cuenta que en la heteroevaluación el docente tiene acceso al documento escrito mientras que los estudiantes no.

\section{Prueba Piloto}

La metodología propuesta se aplicó en la asignatura de Seminario de Investigación, de octavo semestre de ingeniería electromecánica, en la UPTC. Esta asignatura se desarrollaba con la metodología tradicional, en la que el producto final del curso consistía en un anteproyecto sobre una temática propuesta por los estudiantes en grupos de dos personas. La importancia de esta asignatura radica en que los estudiantes ya han superado un $80 \%$ de su formación específica, posibilitando que el estudiante integre los conocimientos disciplinares que ha recibido, en la solución de problemas de ingeniería.

Los resultados descritos en la siguiente sección corresponden a la prueba piloto realizada durante el segundo semestre académico, equivalente a 16 semanas, del año 2012. En el curso se matricularon 24 estudiantes, lo cual permitió conformar grupos de cuatro personas; la conformación de los grupos fue voluntaria y cada uno de ellos escogió, de acuerdo a sus intereses, un problema diferente de los propuestos en el banco de problemas.

\section{RESULTADOS Y DISCUSIÓN}

\section{Ejemplo de aplicación}

El ABP gira alrededor de un problema bien planteado, con una temática motivadora para los estudiantes y cuya solución permita el desarrollo de las competencias que se desean potenciar. A manera de ejemplo se ilustra el enunciado de uno de los problemas, tal y como fue presentado a los estudiantes en la prueba piloto, junto con una breve descripción de la solución brindada por el grupo responsable.

En la vereda Ucuengá de Nobsa, Boyacá, Colombia, las lluvias han venido anegando amplias zonas, tierras con un gran potencial agrícola, conformando un humedal. El problema es que con cada ola invernal el área inundada se incrementa, generando pérdidas a la comunidad. Por lo anterior, es necesario proponer una alternativa, desde el punto de vista técnico, que regule el nivel del humedal e impida su expansión (Correa, 2012). Con el fin de concretar el problema, los estudiantes realizaron una primera toma de contacto con el humedal y con la comunidad afectada por el mismo. Esto permitió identificar el origen del problema: el humedal se localiza en un valle rodeado por montañas, de las cuales fluye agua en época de invierno, la cual se ha venido acumulando en la parte baja del terreno desde hace unos diez años; además, el drenaje natural del agua se ve impedido por el terraplén de una carretera con alto flujo vehicular.

Una primera idea fue desecar el humedal, bombeando el agua hacia un río cercano. Sin embargo, se encontró que, si bien es técnicamente factible, esto no era viable por el daño medioambiental, por el interés de la comunidad en conservar el sitio y por el interés de la administración municipal en utilizarlo como sitio turístico. Además, el dueño del terreno no quería erradicar el humedal, sino evitar que su área continúe creciendo con las siguientes olas invernales. De esta manera los estudiantes identificaron al volumen de 
agua, como la principal variable de tipo técnico. En vista de lo anterior, el grupo optó por proponer el diseño de un sistema que regule el volumen de agua contenida en el humedal, aprovechando para ello los recursos disponibles en el área. En este punto los estudiantes observaron que en los problemas reales, a parte de las variables de tipo técnico, también intervienen aspectos medioambientales, sociales, económicos y políticos, los cuales deben ser tenidos en cuenta a la hora de proponer una solución.

Luego de elaborar un anteproyecto como documento guía para la solución del problema, los estudiantes procedieron a recolectar la información de campo: realizaron una estimación del volumen del humedal, identificaron las facilidades del terreno para instalar un sistema de bombeo y la posible ubicación del cuarto de control, junto con las facilidades para acceder a la red eléctrica. En el informe técnico, la presentación del diseño y cálculo del sistema de bombeo estuvo bien como ejercicio de máquinas hidráulicas, pero faltó justificar las decisiones tomadas en función de las características del humedal y su entorno. Esta situación, común a la mayoría de los grupos, permitió evidenciar la poca relación que establecen los estudiantes entre los conocimientos disciplinares y su aplicación en la solución del problema y su contexto, afectando la toma de decisiones sustentada en la realidad observada, así como la generación de alternativas de solución. Lo anterior debido a que los estudiantes están habituados a resolver problemas cerrados, con solución única, y no a tomar decisiones, algo fundamental en su futuro trabajo como ingenieros, tal y como lo identificaron Mesa y sus colaboradores (2008).

En el informe final los estudiantes presentaron un diseño de los sistemas de bombeo, mando y control, las posibilidades para la alimentación eléctrica y la ubicación de los dispositivos en el terreno. Igualmente elaboraron un presupuesto inicial para implementar la propuesta y la exposición oral se realizó asumiendo que en frente se tenía a la persona con capacidad de decidir la ejecución del proyecto en una fase posterior. Éste ejercicio es importante ya que permite desarrollar destrezas para la presentación de temas técnicos de ingeniería ante un público no experto en la temática, como lo puede ser un administrador, un habitante del sector, el gerente de una empresa o el alcalde de un municipio.

El proyecto del humedal fue muy interesante ya que partió de una problemática concreta del entorno. Además, permitió que los estudiantes tuvieran contacto con la comunidad, recolectaran información de campo y analizaran varias alternativas para la solución del problema. Esta estrategia refleja lo que pueden y deben hacer los estudiantes al enfrentarse a la resolución de un problema real, analizando situaciones y operando sobre ellas, con los conocimientos disciplinares adquiridos y recursos disponibles.

\section{Cambios detectados en las competencias de los estudiantes}

Con el fin de identificar el desarrollo de las competencias profesionales, propuestas en la metodología, se formularon las siguientes preguntas:

\section{P1: ¿Para qué sirve un anteproyecto?}

P2: ¿Qué aspectos se deben tener en cuenta para plantear un problema?

P3: ¿Cuáles son los elementos de un anteproyecto que permiten establecer su coherencia?

P4: ¿Cuáles son los parámetros de evaluación para un anteproyecto?

P5: ¿A lo largo de su formación como ingeniero: ha resuelto problemas reales, del entorno social o industrial, aplicando sus conocimientos de ingeniería?

Tabla 1. Cambios detectados en el desarrollo de competencias

\begin{tabular}{|c|c|c|c|c|c|}
\hline \multirow{3}{*}{ Competencias } & \multirow{3}{*}{$\begin{array}{c}\text { Preguntas } \\
\text { relacionadas con } \\
\text { las competencias }\end{array}$} & \multicolumn{4}{|c|}{ Resultados } \\
\hline & & \multicolumn{2}{|c|}{ Sondeo 1} & \multicolumn{2}{|c|}{ Sondeo 2} \\
\hline & & Incorrecto & Correcto & Incorrecto & Correcto \\
\hline \multirow[t]{2}{*}{ C1 } & $\mathrm{P} 1$ & 10 & 14 & 7 & 17 \\
\hline & P2 & 21 & 3 & 17 & 7 \\
\hline \multirow[t]{2}{*}{$\mathrm{C} 2$} & P3 & 16 & 8 & 8 & 16 \\
\hline & P4 & 24 & 0 & 21 & 4 \\
\hline C3 & P5 & 10 & 14 & 3 & 21 \\
\hline
\end{tabular}


En la Tabla 1 las columnas representan las competencias propuestas en la metodología, las preguntas con las cuales fueron evaluadas y los resultados, dados en número de estudiantes, obtenidos en los sondeos 1 (inicial) y sondeo 2 (final), los cuales se calificaron con los niveles correcto e incorrecto. La C1 se evaluó con las preguntas P1 y P2, C2 se evaluó con las preguntas P3 y P4, mientras que C3 se valoró con P5.

En el sondeo final se evidencian los beneficios de utilizar la metodología ABP en el desarrollo de competencias para estudiantes de ingeniería, teniendo en cuenta que las respuestas correctas aumentaron con respecto a la prueba inicial. Las competencias C1 y C2, relacionadas con la propuesta de solución a problemas del entorno a través del desarrollo de proyectos de ingeniería, mejoraron notablemente en el segundo sondeo, cumpliéndose en buena parte el objetivo de aprendizaje relacionado con la elaboración de un anteproyecto. En este caso la competencia C3, relacionada con la gestión de proyectos de ingeniería a través de la elaboración y socialización de informes, fue la más beneficiada con la aplicación de la metodología propuesta. Sin embargo se observa que un semestre y una materia no son suficientes, ya que el ideal sería que todos los estudiantes alcanzaran un buen nivel en el desarrollo de estas competencias.

\section{Opinión de los estudiantes}

Al indagar a los estudiantes que participaron en la prueba piloto acerca de la conveniencia de la metodología ABP usada durante el semestre, sólo uno la consideró inadecuada ya que, según él, faltó asesoría por parte del tutor para darle completa solución al problema elegido. Los demás estudiantes consideraron que esta metodología es adecuada porque permite potenciar los conocimientos adquiridos en su formación para aplicarlos en el desarrollo del proyecto.

En cuanto a las dificultades para resolver el problema elegido, la falta de recursos técnicos (equipos, instrumentos de medida y materiales) fue el factor más relevante para 10 estudiantes. Nueve estudiantes manifestaron falta de tiempo debido al compromiso con otras asignaturas, mientras que los demás indican dificultades como: Falta de conocimiento en algunos temas, falta de información bibliográfica y discordia entre el grupo. Las respuestas de los estudiantes indican que para una posible implementación de la metodología ABP, la institución deberá tener los recursos técnicos suficientes y permitir el acceso oportuno para que los estudiantes aborden eficientemente la búsqueda a la solución de los problemas. Igualmente, se sugiere que se relacione el tema del problema propuesto con las asignaturas afines, con el fin de realizar un trabajo paralelo y así disminuir el problema de tiempo por compromisos con otras asignaturas.

\section{Opinión del docente}

El docente manifiesta que todas las etapas son importantes, pero la más crítica es el anteproyecto, ya que en él se delimita el problema y se establecen los alcances del proyecto. De no hacer una buena labor en este punto, es bastante difícil que se llegue a un buen fin. La motivación que se hace es fundamental, especialmente al principio del proceso, con el fin de clarificar la metodología, los roles y los productos esperados en cada etapa. En el caso de la prueba piloto fue difícil vencer la inercia ya que los estudiantes son de octavo semestre y han sido formados con la metodología tradicional.

A la pregunta: ¿Cuáles fueron los problemas que, en su opinión, afectaron la prueba piloto? Aunque al formular los temas se tuvo en cuenta el nivel de formación de los estudiantes, fue evidente la falta de conocimientos relacionados con electrónica, especialmente con microcontroladores, adquisición y procesamiento de datos, al igual que con el uso de simuladores. En cuanto a los recursos, el acceso a los mismos fue una limitante. Si bien la mayoría de los elementos están en la institución, los estudiantes no siempre pueden acceder a ellos por incompatibilidad en los horarios. Otro problema es el tiempo extra clase que deben dedicar los estudiantes al desarrollo del proyecto. En muchas ocasiones es un inconveniente debido al cruce de horarios y a la dispersión de semestres en que se encuentran matriculados.

A la pregunta: ¿Cuáles son las ventajas y desventajas de la metodología como estrategia para el desarrollo de competencias profesionales en el Ing. Electromecánico?, el docente destaca como ventajas que: Permite a los estudiantes aplicar los conocimientos previos, mejorando las destrezas en el uso de técnicas como la manipulación de instrumentos y el manejo de software. Mejora la competencia de trabajo en grupo, pues el resultado final depende del aporte que hacen todos los integrantes. Debido a la multiplicidad de tareas, es necesario que los estudiantes compartan información y asuman responsabilidades, competencias bastante difíciles de alcanzar con otras metodologías.

Entre las desventajas se tiene que es complicado evaluar el aporte individual, pues las actividades están enfocadas a potenciar el trabajo en grupo y al desarrollo de competencias que implican un alto grado de interactividad. Además, al trabajar diferentes temáticas, es difícil para un solo docente brindar la asesoría, desde el punto de vista técnico, en todas ellas. Como recomendaciones para mejorar la metodología, el 
docente plantea que ésta podría emplearse de manera coordinada en varias asignaturas a lo largo de la carrera, conformando un equipo de docentes que seleccionen los temas, realicen las asesorías y la evaluación. De esta manera se podría potenciar el proceso formativo de los estudiantes, junto con el trabajo en grupo de los docentes. Disponer de un espacio académico, a manera de evento, que permita visibilizar los logros alcanzados sería un factor adicional de motivación para los estudiantes, ya que les permitiría presentar sus proyectos a la comunidad académica del programa. En este sentido, algunas instituciones han cambiado sus planes de estudio originalmente concebidos por objetivos, a planes de estudio basados en competencias (Schmal, 2012), asumiendo los retos que ello implica: la construcción de un marco teórico que de sustento al nuevo modelo, el ajuste de los procesos administrativos, la reorientación de los roles que cumplen docentes y estudiantes, junto con la modificación del perfil del egresado para adecuarlo a las exigencias del contexto.

\section{CONCLUSIONES}

Proponer y gestionar proyectos de ingeniería, que respondan a necesidades culturales y sociales de contextos específicos, es una de las principales competencias para los ingenieros del presente milenio. En este sentido, la prueba piloto permitió establecer el avance de los estudiantes en el desarrollo de competencias profesionales propias de la ingeniería, gracias a la metodología propuesta.

Para aplicar el ABP en el desarrollo de competencias profesionales en ingeniería, se propuso una metodología que implica determinar el papel que juegan docentes y estudiantes, las competencias a ser abordadas en el proceso formativo, los puntos de control y los mecanismos de evaluación.

Las temáticas se eligieron de acuerdo con el nivel de formación de los estudiantes, de modo que pudieran aplicar los conocimientos adquiridos previamente. En la prueba piloto se detectaron falencias en el área de electrónica; también se detectó falta de habilidad en el uso de programas de simulación así como poca experiencia en el uso de algunos instrumentos de medida.

Lo anterior permite establecer que el ABP, aparte de ser una estrategia de aprendizaje, puede ser utilizado como una herramienta para diagnosticar y corregir las debilidades en la formación de los futuros ingenieros.

Para alcanzar mejores resultados, esta metodología debería ser aplicada de forma institucional, a lo largo del plan de estudios. Si bien un esfuerzo aislado como el de la prueba piloto permite identificar debilidades y fortalezas en la formación de los futuros ingenieros, éstas no podrán ser corregidas o potenciadas en una sola asignatura, sino a través del esfuerzo conjunto del colectivo docente.

\section{REFERENCIAS}

Alejandro, C., Prácticas de Laboratorio de Física General en Internet, REEC: Revista Electrónica de Enseñanza de las Ciencias, ISSN-e: 1579-1513 (en línea), 3(3), 202-210 (2004). http://reec.uvigo.es/ volúmenes/volumen3/REEC_3_2_6.pdf. Acceso: 5 de Enero (2013).

Benítez, A. y M. García, Un Primer Acercamiento al Docente Frente a una Metodología Basada en Proyectos, Form. Univ., ISSN-e: $0718-5006$ (en línea), 6(1), 21-28 (2013).

Correa, M., El Aprendizaje Basado en Problemas Como Estrategia Para la Formación de Ingenieros Electromecánicos, Pregrado, Universidad Pedagógica y Tecnológica de Colombia, Facultad Seccional Duitama, (2012).

Domínguez, J., E. Carod y M. Velilla, Comparativa Entre el Aprendizaje Basado en Proyectos y el Aprendizaje Basado en Problemas. II Jornadas de Innovación Docente, Tecnologías de la Información y de la Comunicación e Investigación Educativa en la Universidad de Zaragoza, ISSN: 1330-1012 (en línea), (2008). http://cmapspublic2.ihmc.us/rid=1J9HKH72N9B9GQGT9F/Informaci\%C3\%B3n\%20adjunta\%203.pdf. Acceso: 5 de Febrero (2013).

Echazarreta, D. y R. Haudemand, Resolución de Problemas Integradores en la Enseñanza de la Física para Estudiantes de Ingeniería Civil. Form. Univ., 2(6), 31-38 (2009).

Ertugrul, N. Towards Virtual Laboratories: A survey of LabView-Based Teaching/Learning Tools and Future Trends . International Journal of Engineering Education, 16, 171-180 (2000).

Galvis, R. V., De un Perfil Docente Tradicional a un Perfil Docente Basado en Competencias, Acción Pedagógica, 16(1), 48-57 (2007).

González, M. y I. Ramírez, La formación de competencias profesionales: un reto en los proyectos curriculares universitarios. Odiseo, Revista Electrónica de Pedagogía, 8(16), (2011). 
González, V., ¿Qué significa ser un Profesional Competente? Reflexiones Desde una Perspectiva Psicológica. Revista Cubana de Educación Superior. 22(1), 45-53 (2002).

Hernández, C., Utilización del Trabajo por Proyectos para Incentivar la Innovación Tecnológica en los Estudiantes Universitarios. Revista Científica de la Fundación Iberoamericana para la Excelencia Educativa Hecademus, 3(8), 42-54 (2010).

Iglesias, J., El aprendizaje Basado en Problemas en la Formación Inicial de Docentes. Perspectivas, 32(3) (2002).

ITESM, Inst. Tecnol. y de Estudios Superiores de Monterrey. (2000). El Aprendizaje Basado en Problemas como Técnica Didáctica. Dirección de Investigación y Desarrollo Educativo, Vicerrectoría Académica http://www.rsu.uninter.edu.mx/doc/marco_conceptual/EIMetododeProyectoscomoTecnicaDidactica.pdf.

Acceso: 9 de Abril (2012).

Kurt, S. y A. Ayas, Improving Students Understanding and Explaining Real Life Problems on Concepts of Reaction Rate by Using a Four Step Constructivist Approach. Energy Education Science and Technology Part B: Social Educational Studies, 4(2), 979-992 (2012). 1308-7711

Lehmanna, M., P. Christensen a, X. Dua y M. Thranea, Problem-oriented and Problem-Based Learning (POPBL) as an innovative learning strategy for Sustainable Development in Engineering Education, European J. Engineering Education, 33(3), 283-295 (2008).

Mesa, J., J. Álvarez, J. Villanueva y F. de Cos, Actualización de Métodos de Enseñanza Aprendizaje en Asignaturas de Dirección de Proyectos de Ingeniería. Form. Univ., 1(4), 23-28 (2008).

Moreno, L., C. González, I. Castilla, E. González y J. Sigut, Applying a Constructivist and Collaborative Metodological Approach in Engineering Education, Computers and Education, 49(1), 891-915 (2007).

Nascimento, J. M. y Amaral E. M., O Papel das interações sociais e de atividades propostas para o ensinoaprendizagem de conceitos químicos, Ciência \& Educação, 18(3), 575-592 (2012).

Okutsu, M., D. DeLaurentis, S. Brophy y J. Lambert, Teaching and Aerospace Engineering Design Course via Virtual Worlds: A Comparative Assessment of Learning Outcomes. Computers and Education, 60(1), 288-298 (2013).

Painean, O., V. Aliaga y T. Torres, Aprendizaje Basado en Problemas: Evaluación de una Propuesta Curricular Para la Formación Inicial Docente, Estud. pedagóg., ISSN: 0718-0705 [en línea], 38(1), 161-180 (2012). http://www.scielo.cl/scielo.php?script=sci_arttext\&pid=S0718-07052012000100010\&lng=es\&nrm=iso. Acceso: 3 de Febrero (2013).

Regalado, A., M. Cid y J. Báez, Problem based learning (PBL) : Analisys of continuous stirred tank chemical reactors with a process control approach. International Journal of Software Engineering \& Applications (IJSEA), 1(4), 54-73 (2010).

Regalado, A., E. Peralta y J. Báez, Aprendizaje Basado en Competencias Aplicado a una Asignatura de Transferencia de Calor. Form. Univ., 4(1), 13-18 (2011).

Restrepo, B., Aprendizaje Basado en Problemas (ABP): Una Innovación Didáctica para la Enseñanza Universitaria. Educación y Educadores, 8, (2005).

Rosado, A., M. Bataller y J. Guerrero, Aprendizaje por Proyectos: Una Aproximación Docente al Diseño Digital Basado en VHDL. IEEE - RITA, 3(2), 88-95 (2008).

Rugarcia, A., R.M. Felder, D.R. Woods y J.E. Stice, The Future of Engineering Education I. A Vision for a New Century," Chem. Eng. Ed., 34 (1), 16-25 (2000).

Schmal, R., Reflexiones en Torno a un Programa para la Formación de Competencias Transversales en Ingeniería, Ciencia, Docencia y Tecnología, 44(1), 239-262 (2012).

Tobón, S. y A. Jayk, Experiencias de Aplicación de las Competencias en la Educación y el Mundo Organizacional. Editor: Red Durango de Investigadores Educativos, Primera Edición, México (2012).

Vacca, V., E. Caicedo y J. Ramírez, Herramienta Remota de Cálculo y Multiusuario para el Aprendizaje Basado en Problemas Usando Matlab. Revista Facultad de Ingeniería Universidad de Antioquia, (59), 158169 (2011). 
